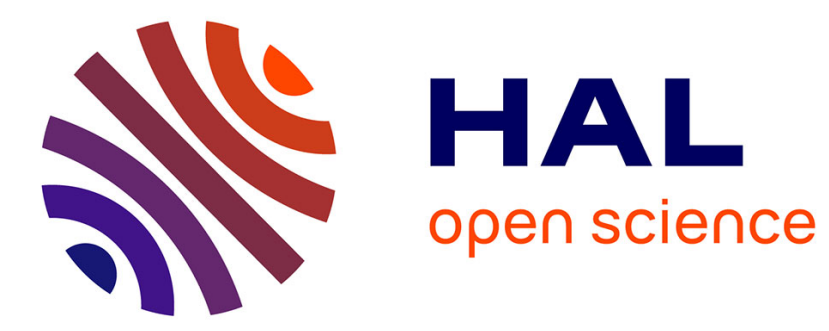

\title{
Hepatic manifestations of cystic fibrosis
}

Jeremy Dana, Muriel Girard, Dominique Debray

\section{To cite this version:}

Jeremy Dana, Muriel Girard, Dominique Debray. Hepatic manifestations of cystic fibrosis. Current Opinion in Gastroenterology, 2020, 36 (3), pp.192-198. 10.1097/MOG.0000000000000624 . hal02881071

\section{HAL Id: hal-02881071 \\ https://hal.sorbonne-universite.fr/hal-02881071}

Submitted on 25 Jun 2020

HAL is a multi-disciplinary open access archive for the deposit and dissemination of scientific research documents, whether they are published or not. The documents may come from teaching and research institutions in France or abroad, or from public or private research centers.
L'archive ouverte pluridisciplinaire HAL, est destinée au dépôt et à la diffusion de documents scientifiques de niveau recherche, publiés ou non, émanant des établissements d'enseignement et de recherche français ou étrangers, des laboratoires publics ou privés. 


\section{Current Opinion in Gastroenterology Hepatic manifestations of Cystic fibrosis \\ --Manuscript Draft--}

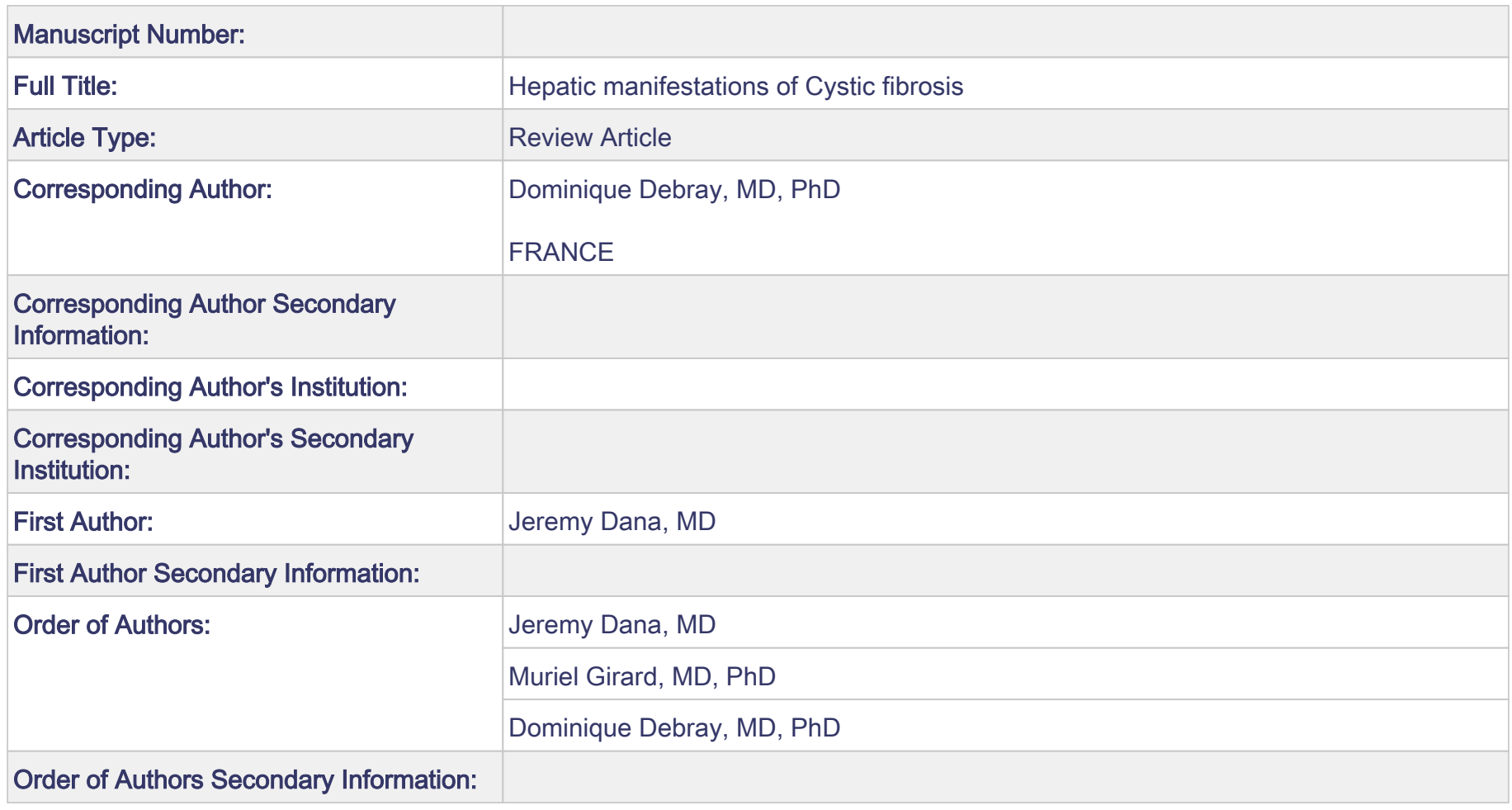




\section{Hepatic manifestations of Cystic fibrosis}

Jeremy Dana, MD ${ }^{1}$, Muriel Girard, $\mathrm{MD}, \mathrm{PhD}^{2,3}$, Dominique Debray, $\mathrm{MD}, \mathrm{PhD}^{2,4}$.

${ }^{1}$ Department of Pediatric Radiology, APHP-Necker Hospital, Paris, France

${ }^{2}$ Pediatric Hepatology Unit, Reference center for Biliary Atresia and Cholestatic genetic diseases, APHP-Hôpital Necker, Paris, France

${ }^{3}$ Laboratory of Nutrient Sensing Mechanisms,, Inserm U1151, Institut Necker Enfants Malades

(INEM), Université de Paris

${ }^{4}$ Sorbonne Université, INSERM, Centre de Recherche Saint-Antoine (CRSA), Paris, France

\section{Corresponding author}

Dominique Debray, $\mathrm{MD}, \mathrm{PhD}$

Pediatric Hepatology unit

Hôpital Necker-Enfants Malades, 149 rue de SEVRES, 75015 PARIS, France

Tel : 331444925 88; Fax : 33144492501

Email : dominique.debray@aphp.fr

Conflicts of interest : none

Financial support and sponsorship : none.

Word counts: Abstract : 197 words ; Text : 2603 words

List of abbreviations: ALT, alanine aminotransferase ; AST, aspartate aminotransferase ; APRI, AST to platelet ratio index; AUC, area under the curve; FIB-4, Fibrosis-4 index; CF, cystic fibrosis ; CFTR, cystic fibrosis transmembrane conductance regulator; CF-LD, CF-related liver disease; GGT, gamma-glutamyl transferase; HVPG, hepatic venous pressure gradient; MRE, magnetic resonance elastography; SWE, shear wave elastography; TE, transient elastography ; US, ultrasonography, 


\begin{abstract}
Purpose of review: Liver disease in CF usually develops before puberty, is often asymptomatic and slowly progressive. Multilobular cirrhosis develops in approximately 5-10\% of patients by the age of 18 , and is a significant contributor to the morbidity and mortality. No therapy, including ursodeoxycholic acid and CFTR correctors or potentiators, has proven effective to prevent or halt the progression of liver disease towards cirrhosis and portal hypertension. This review provides the current knowledge in the epidemiology of CF liver disease and development of non-invasive tools to assess liver disease severity and progression overtime in order to optimise clinical management and therapeutic options.
\end{abstract}

Recent findings: Liver disease develops during childhood but also later in the lifetime of patients with $\mathrm{CF}$; the incidence of cirrhosis with portal hypertension increases progressively reaching $10 \%$ by age 30. Several non-invasive tools to measure liver stiffness as an indirect measure of fibrosis are being investigated, and show promising results for the assessment of early stages of liver fibrosis and disease progression.

Summary: Identifying non-invasive biomarkers is fundamental to improving early diagnosis, monitoring disease evolution and measuring treatment effects. A pre-requisite is the use of consistent definitions for CF-LD in clinical trials.

Keywords: Cystic fibrosis, liver disease, portal hypertension, epidemiology, elastography 


\section{Introduction}

Cystic fibrosis (CF; OMIM 219700), is the most common autosomal recessive disease of the Caucasian population, caused by pathogenic variants in the gene encoding the cystic fibrosis transmembrane conductance regulator (CFTR), a chloride channel expressed in epithelial cells. It is a multiorgan disease affecting mostly the lungs, pancreas, sweat glands, intestine and the liver. Liver involvement in CF shows considerable variability, including hepatobiliary involvement (such as focal biliary fibrosis, multilobular cirrhosis, large duct biliary strictures, microgallbadder, gallbladder dyskinesia, cholelithiasis), obliterative portal venopathy, steatosis and other liver abnormalities resulting from infections, long-term antibiotic therapy, diabetes, nutritional deficiencies, hypoxemia, hepatic congestion from right-side heart failure (1-5). Multilobular cirrhosis has emerged as a significant contributor to the morbidity and mortality, being the third cause of death after respiratory failure and transplantation-related complications in patients with CF (6). No therapy, including ursodeoxycholic acid and CFTR correctors or potentiators, has proven effective to prevent or halt the progression of liver disease towards cirrhosis and portal hypertension (7).

Progress in the understanding of relevant mechanisms of hepatobiliary disease will hopefully optimise future therapeutic options to prevent or halt the progression of fibrosis towards multilobular cirrhosis. Studies, recently reviewed by Debray et al (8) and Fiorotto et al (9), support the view that cholangiopathy arising in $\mathrm{CF}$ is the result of an ill-adapted innate immune response to endotoxins coming from the intestine and triggering a pro-inflammatory response leading to peribiliary inflammation and fibrosis. We also recently showed in CF mouse models, that the development of biliary disease required the combination of CFTR defect with both genetic and environmental factors such as diet-induced dysbiosis (10). In subjects bearing the SERPINA1 Z alleles, who are at increased risk of developing cirrhosis and portal hypertension, one may speculate that the accumulation of alpha-1-antitrypsin variant in cholangiocytes induces an endoplasmic reticulum stress as it does in hepatocytes, and in conjonction with the endoplasmic reticulum stress induced by the accumulation 
of F508del CFTR, predispose cholangiocytes to injury of other causes, e.g. endotoxins or toxic bile $(8,11)$.

The need for a consistent definition of CF related LD (CF-LD) is also essential for addressing epidemiology, natural course and optimise future therapeutic options (12). A phenotypic characterization of the liver involvement, outlined in Debray et al (12), appears as the most pertinent for clarity in reporting for epidemiological purposes, evaluation of diagnostic tools such as elastography, long-term outcomes, and selection of patients for inclusion in therapeutic studies.

This review provides the current knowledge in the epidemiology and outcome of liver disease in CF and advances in the development of non-invasive tools to assess liver disease severity and progression overtime in order to optimise clinical management and evaluate future therapeutic options.

\section{Epidemiology}

The prevalence of CF-LD remains uncertain since diagnostic criteria used to define CF-LD do not differentiate CFTR related hepatobiliary disease from other liver involvement not related to the CFTR defect (12). Indeed, in most epidemiological studies, CF-LD was defined, according to the European criteria, when at least two of the following characteristics were present: (1) abnormal physical examination; (2) abnormalities of liver function tests; (3) evidence of liver involvement at ultrasonography (US) (21).

Historical long-term follow-up studies reported a cumulative incidence of liver involvement increasing through childhood and reaching a plateau in mid-adolescence ranging between $27 \%$ and $41 \%$, without incident cases after the age of 18 . The progression of liver fibrosis to cirrhosis with portal hypertension was reported in a minority of patients (5 to 10\%) at a mean age at diagnosis of 10 years $(6,13-15)$. Although most patients present in childhood, recent studies suggest the occurence of liver disease in adulthood and the need for lifelong screening for liver disease $(13,16)$. Based on a large cohort of $3328 \mathrm{CF}$ patients with pancreatic insufficiency recruited into the French CF Modifier Gene Study since 2004, the cumulative incidence of liver involvement, defined according to the 
European criteria (17), was found to increase by approximately $1 \%$ every year, reaching $32.2 \%$ by age 25 (13). The incidence of severe CF-LD (i.e cirrhosis and/or portal hypertension) increased only after the age of 5 , reaching $10 \%$ by age 30 (13). Using the same cohort, the cumulative incidence of CF-LD was shown to increased more rapidly in patients carrying the SERPINA1 $\mathrm{Z}$ allele (3\%) reaching $47 \%$ by age 25 compared with $30 \%$ in noncarriers (11). Increased risk was similar for patients with severe $\mathrm{CF}-\mathrm{LD}(\mathrm{HR}=1.5,95 \% \mathrm{CI}=0.7-3.2, \mathrm{P}=0.31)$ but failed to reach significance due to a limited sample size of Z-allele carriers (11). Another longitudinal cohort study including 577 CF patients diagnosed by newborn screening confirmed a prevalence of portal hypertension of about $9 \%$ with an incidence rate of near 3/1000 patient years in patients 3-18 years of age (15).

\section{Outcome of liver disease}

The most clinically significant liver disease is multilobular cirrhosis which most commonly manifests as complications of portal hypertension in childhood $(14,15)$. Recently, both the 6 -year survival rate (77 vs. $93 \% ; \mathrm{P}<.01)$ and the median age at death (27 vs. 37 years; $\mathrm{P}=0.02$ ) were shown to be significantly lower in cirrhotic $(n=95)$ compared to non cirrhotic CF controls (6). The reported primary cause of death was pulmonary in $68 \%$ of cirrhotic cases, and liver failure related in $18 \%$ of cases. In another recent longitudinal birth cohort study of $577 \mathrm{CF}$ patients, 51 of whom developed portal hypertension, combined mortality-liver transplant rate was significantly increased in patients with portal hypertension versus those without portal hypertension $(23.5 \%$ vs $4.8 \%$; $<<0.001)(15)$.

Because portal hypertension may develop in CF patients without cirrhosis (i.e non cirrhotic portal hypertension related to obliterative portal venopathy) (3-5), extreme care should be taken not to underestimate the degree of portal hypertension, even if there is little evidence of liver disease clinically or on imaging.

There is no available evidence of the benefit of primary prophylactic interventions to prevent bleeding from esophageal varices in terms of safety, efficacy and survival outcomes (12). In a recent large study including 943 participants (mean age 18.1 years) with reported cirrhosis in the CF Foundation 
Patient Registry from 2003 to 2012, ten-year cumulative variceal bleeding, liver transplantation and liver death rates were $6.6 \%, 9.9 \%$ and $6.9 \%$ respectively, with an all-cause mortality of $39.2 \%$; allcause mortality was not increased in subjects with variceal bleeding compared to those without (18). If esophageal variceal bleeding occurs, endoscopic band ligation is successful in most cases. Additional therapeutic interventions may be required in patients with intractable esophageal bleeding, recurrent bleeding from gastric or rectal varices and portal hypertensive gastropathy. Studies are lacking to identify the most beneficial and least risky therapeutic option, and the appropriate timing of intervention $(2,12)$. Transjugular intrahepatic portosystemic and surgical shunting procedures have both been successfully employed for portal decompression in CF patients with recurrent bleeding, both as a long-term therapy for portal hypertension or as a bridge to liver transplantation $(2,19)$. Recommandations for liver transplantation evaluation have been recently established (20). Those who develop hepatic decompensation or complications of portal hypertension such as refractory ascites, recurrent variceal bleeding not controlled by endoscopic management. hepatopulmonary syndrome/portopulmonary hypertension, may benefit from liver transplant, either alone, or in combination with lung transplant (20). In view of the good hepatic synthetic function, management of patients with non cirrhotic portal hypertension should probably seek the alleviation of this portal hypertension by shunting procedures rather than referring these patients for liver transplantation.

\section{Biomarkers to assess the presence of liver disease}

With its frequently asymptomatic presentation, liver disease can be very difficult to diagnose in the early phases of development. Several methods have shown promise for the detection of clinically silent liver disease and are being evaluated among the CF population.

Serum biomarkers. 
Liver enzyme abnormalities lack specificity and sensitivity for the diagnosis of liver disease. They may result from drug hepatotoxicity, infection, steatosis or any other cause of concommittant acute or chronic liver disease that needs to be ruled out (17). Persisting increased serum levels of gammaglutamyl transferase (GGT) is the only biochemical feature that may predict the progression of hepatobiliary disease towards cirrhosis $(15,21,22)$.

When compared to histology, the aspartate aminotransferase (AST) to Platelet Ratio Index (APRI) and Fibrosis-4 (FIB-4 ) index that incorporate standard laboratory data (i.e. AST, ALT, platelet count, age) show a good correlation with advanced fibrosis (i.e cirrhosis), but not for differentiating earlier stages of fibrosis (23). When compared to liver US, Ling et al. show an excellent discriminating ability for nodular liver versus normal liver in a multivariable model including GGT and APRI $($ AUROC $=96 \%, 95 \%$ CI:0,92-1) (24). This result is strengthened by Sellers et al who showed that combining GGT, APRI and GGT to platelet ratio allowed to identify correctly $85 \%$ of nodular livers with high sensitivity (93.0\%) and negative predictive value (98.2\%). These biomarkers may help identify which CF children should undergo US evaluation. However, there is still a lack of sensitive serum biomarkers to predict or detect fibrosis at early stages.

\section{Imaging biomarkers}

Liver US remains the most suitable initial method of investigation for assessment of abnormalities of the liver parenchyma, and of signs of portal hypertension (17, 24-26). Abnormal echogenicity may precede clinical manifestations of liver disease, and may identify patients at risk of progressive disease (24-26). Sellers et al. recently highlighted the importance of US as a routine exam and valuable marker of early liver disease in CF (26). Among 82 patients (88\% with no clinical/biochemical signs of liver involvement), only $52 \%$ had a normal liver US, $29 \%$ had a hyperechogenicity pattern and above all $18 \%$ had a nodular liver. However, US has a few limitations: there is significant intra- and inter-observer variability, hyperechogenicity and/or heterogeneity can 
be related to steatosis or fibrosis and a normal ultrasound doesn't rule out the existence of extensive fibrosis (27).

Magnetic resonance (MR) imaging, although costly, is particularly interesting by allowing an exhaustive assessment of the liver parenchyma, nodule, steatosis, portal hypertension and biliary tree. MR- cholangiography allows a high diagnostic performance in detecting biliary focal stenosis (Figure 1) in patients with or without clinically apparent liver disease or liver test abnormalities (1). Furthermore, the severity of steatosis can be accurately assessed by MR imaging proton density fat fraction (Figure 2). This sequence has an excellent diagnostic performance to assess quantitatively hepatic fat content and an excellent correlation with histologic grades (28).

\section{Measurement of liver stiffness by elastography}

Because liver biopsy is an imperfect gold standard (invasive, risk of bleeding, may underestimate the degree of focal fibrosis, costly), non-invasive assessment may help to diagnose and follow the progression of liver fibrosis. Several clinical ultrasonographic systems, such as transient elastography

(TE), point or supersonic shear-wave elastography (SWE), and magnetic resonance elastography (MRE) are currently available for quantitative elastography measurements of liver stifness as an indirect measure of fibrosis in clinical practice but better-quality studies and further validation are still needed in CF, particularly for the diagnosis of mild to moderate fibrosis.

Transient Elastography (TE) appears to be well correlated with severe fibrosis and can be combined with APRI to improve the differentiation of CF patients with F3-F4 vs F1-F2 fibrosis (27, 29). TE was also shown to be a reliable method to assess liver disease progression $(30,31)$. However, TE performances are limited by peri-hepatic ascite, obesity and steatosis.

Shearwave Elastography (SWE) is a fast, simple and reliable method performed in real time during a conventional US examination allowing targeted measurements. The diagnostic accuracy of SWE for assessing liver fibrosis in children, even early stage (F1-F2) fibrosis, was reported in a series of 45 children (age range from 1 month to 17 years) with various liver diseases (but no CF-LD) who 
underwent a liver biopsy (32). According to the degree of liver fibrosis at liver biopsy, 88.5\%-96.8\% of patients were correctly classified, with areas under the curve (AUC) of 0.90-0.98 (95\% confidence interval $[\mathrm{CI}]: 0.8,1.0)$. The AUC for patients with stage F0 versus stage F1-F2 was 0.93 (95\% CI: 0.87, 0.99)(32). Calvopina et al. conducted the only study in children with cystic fibrosis and also showed that SWE is an accurate technique in detecting and assessing liver fibrosis. Diagnostic accuracy was improved when combined with APRI (33).

Finally, MR- Elastography (MRE) is the latest technique that uses low-frequency external mechanical wave emission and reconstruction algorithms to obtain a color mapping of liver elasticity. It allows early detection, quantification and localization of patchy fibrosis lesions (Figure 3). It can be performed in a single breath hold by using a spin-echo echo-planar imaging (SE-EPI), very useful in young children. If steatosis is one of the main aspects of liver involvement in $\mathrm{CF}$, it does not affect measurement of liver stiffness (34). Schwimmer showed in a recent study that MRE had a good diagnostic performance in the evaluation of liver fibrosis in children with non-alcoholic fatty liver disease versus histopathology (35). Finally, when compared to liver histology, MRE was shown to provide a higher diagnostic accuracy than TE and SWE for staging early stages of liver fibrosis in adults with chronic liver disease caused by hepatitis B or C virus, nonalcoholic fatty liver disease, or autoimmune hepatitis (36).

To conclude, TE, SWE and MRE seem to exhibit comparable and very good to excellent diagnostic accuracy for advanced fibrosis (37). In our opinion, the continuous assessment of liver stiffness may become the new gold standard for the diagnosis of progressive fibrosis, to be implemented in therapeutic intervention studies. Although accurate cut-offs in CF remain to be determined, SWE may be more accurate than TE for assessing early stages of fibrosis, mainly due to the focal nature of fibrosis. MRE is also a promising, but costly, procedure for assessing early stages of liver fibrosis in CF patients. 


\section{Biomlarkers to assess the presence of portal hypertension}

Upper gastrointestinal endoscopy remains the most accurate and reliable test to detect the presence of esophageal varices and portal hypertensive gastropathy, and most centres offer endoscopic screening and treatment of large varices (12).

However, esophageal capsule endoscopy for the evaluation of esophageal or small bowel varices is being evaluated in children and would be particurlarly useful in the CF population to avoid deleterious repeated general anesthesia $(38,39)$.

Spleen stiffness as measured by TE or SWE was shown to have a good diagnostic value to predict esophageal varices in children with various chronic liver diseases (40-43). Spleen stiffness combined with non-invasive biomarkers such as platelet counts and spleen size may improve the bleeding prediction $(44,45)$.

Hemodynamic measurements of hepatic venous pressure gradient may be indicated in patients with portal hypertension but without evidence of cirrhosis to measure the hepatic venous pressure gradient (HVPG). Intrahepatic presinusoidal (non-cirrhotic) portal hypertension is indicated if the HVPG is normal $(\leq 5 \mathrm{mmHg})$ or only slightly increased $(5-10 \mathrm{mmHg})(3,5)$

In conclusion, multilobular cirrhosis is the most significant CF-hepatobiliary complication that impacts clinical outcomes. While liver disease develops most often in the pediatric age, recent epidemiological data show that the incidence of liver disease continuously increase over time, with a consequential rate of progression to severity. Challenges for the future are to develop novel therapies to prevent or halt the progression of the disease. Refining existing non-invasive biomarkers and identifying additional markers is fundamental to improving diagnosis, monitoring disease evolution and measuring treatment effects. Several non-invasive tools to assess liver fibrosis are being investigated in $\mathrm{CF}$, and show promise for the detection of clinically silent liver disease. A consensus definition and classification of CF-LD among all CF centers is however essential for assessing effects 
of novel therapeutic strategies, including CFTR correctors and potentiators. An agreement between North American and European associations is expected this year.

\section{Key points}

- Liver disease develops progressively in the lifetime of patients with $\mathrm{CF}$ with a cumulative incidence of cirrhosis and portal hypertension of $10 \%$ by age 30 .

- Several non-invasive tools to measure liver stiffness as an indirect measure of fibrosis show promising results for the assessment of early stages of liver fibrosis and progression overtime.

- The use of a phenotypic characterization of the liver involvement in CF appears as the most pertinent for clarity in reporting for epidemiological purposes, evaluation of diagnostic tools such as elastography, long-term outcomes, and selection of patients for inclusion in therapeutic studies.

\section{References and recommended reading}

Papers of particular interest, published within the annual period of review, have been highlighted as:

* of special interest

** of outstanding interest

1. Assis DN, Debray D. Gallbladder and bile duct disease in Cystic Fibrosis. J Cyst Fibros 2017;16 Suppl 2:S62-S69.

2*. Debray D. Liver disease in Cystic Fibrosis. In: Pediatric Hepatology and Liver Transplantation, edition 1, D'Antiga Lorenzo (Ed.). Springer Nature Switzerland AG; Springer International Publishing, 2019: pp.255-269.

This book chapter provides the reader with a complete review on clinical aspects, diagnostic tools, and management of liver disease in cystic fibrosis.

3. Witters P, Libbrecht L, Roskams T, et al. Liver disease in cystic fibrosis presents as noncirrhotic portal hypertension. J Cyst Fibros 2017;16:e11-e13. 
4. Hillaire S, Cazals-Hatem D, Bruno O, et al. Liver transplantation in adult cystic fibrosis: Clinical, imaging, and pathological evidence of obliterative portal venopathy. Liver Transpl 2017;23:1342-1347.

5*. Wu H, Vu M, Dhingra S, et al. Obliterative Portal Venopathy Without Cirrhosis Is Prevalent in Pediatric Cystic Fibrosis Liver Disease With Portal Hypertension. Clin Gastroenterol Hepatol 2019;17:2134-2136.

The authors report the clinical features and liver pathology in 17 explants from children and adolescents with CF who underwent liver transplantation. Nodular regenerative hyperplasia without cirrhosis was seen in $94 \%$ of explants with evidence for obliterative portal veinopathy in 11 $(64.7 \%)$.

6*. Pals FH, Verkade HJ, Gulmans VAM, et al. Cirrhosis associated with decreased survival and a 10-year lower median age at death of cystic fibrosis patients in the Netherlands. J Cyst Fibros 2019;18:385-389.

This study demonstrates that the development of cirrhosis impacts clinical outcomes, the primary cause of death being pulmonary in $68 \%$ of cases.

7. Cheng K, Ashby D, Smyth RL. Ursodeoxycholic acid for cystic fibrosis-related liver disease. Cochrane Database Syst Rev 2017;9:CD000222.

8*. Debray D, Corvol H, Housset C. Modifier genes in cystic fibrosis-related liver disease. Curr Opin Gastroenterol 2019;35:88-92.

This review provides the latest knowledge in the pathophysiology of CFLD and CF genetic modifier research in CFLD.

9**. Fiorotto R, Strazzabosco M. Pathophysiology of Cystic Fibrosis Liver Disease: A Channelopathy Leading to Alterations in Innate Immunity and in Microbiota. Cell Mol Gastroenterol Hepatol 2019;8:197-207.

This review provides an overview of the current understanding of the pathophysiology of the disease and discusses a potential target for intervention. 
10*. Debray D, El Mourabit H, Merabtene F, et al. Diet-Induced Dysbiosis and Genetic Background Synergize With Cystic Fibrosis Transmembrane Conductance Regulator Deficiency to Promote Cholangiopathy in Mice. Hepatol Commun 2018;2:1533-1549.

This experimental study in CF mice provides evidence that CFTR deficiency causes abnormal intestinal permeability, which, combined with diet-induced dysbiosis and immune-related genetic susceptibility, promotes CF-related cholangiopathy.

11*. Boelle PY, Debray D, Guillot L, et al. SERPINA1 Z allele is associated with cystic fibrosis liver disease. Genet Med 2019;21:2151-2155.

This database study describes the increased incidence of liver disease and severe liver disease in SERPINA1 Z allele carriers compared to non carriers.

12. Debray D, Narkewicz MR, Bodewes F, et al. Cystic Fibrosis-related Liver Disease: Research Challenges and Future Perspectives. J Pediatr Gastroenterol Nutr 2017;65:443-448.

13*. Boelle PY, Debray D, Guillot L, et al. Cystic Fibrosis Liver Disease: Outcomes and Risk Factors in a Large Cohort of French Patients. Hepatology 2019;69:1648-1656.

This large database study including 3,328 patients with CF describes the incidence of liver disease and the impact of ursodeoxycholic acid therapy on the incidence of severe liver disease.

14. Stonebraker JR, Ooi CY, Pace RG, et al. Features of Severe Liver Disease With Portal Hypertension in Patients With Cystic Fibrosis. Clin Gastroenterol Hepatol 2016;14:1207-1215 e1203. 15*. Marco C, Judith F, Donna W, et al. Occurrence, outcomes and predictors of portal hypertension in cystic fibrosis: A longitudinal prospective birth cohort study. J Cyst Fibros 2019. This is the first cohort study to assess the prevalence (of about 9\%), incidence (of 3/1000 patient years) of portal hypertension and combined mortality transplant rate associated with portal hypertension.

16. Koh C, Sakiani S, Surana P, et al. Adult-onset cystic fibrosis liver disease: Diagnosis and characterization of an underappreciated entity. Hepatology 2017;66:591-601. 
17. Debray D, Kelly D, Houwen R, et al. Best practice guidance for the diagnosis and management of cystic fibrosis-associated liver disease. J Cyst Fibros 2011;10 Suppl 2:S29-36.

18. Ye W, Narkewicz MR, Leung DH, et al. Variceal Hemorrhage and Adverse Liver Outcomes in Patients With Cystic Fibrosis Cirrhosis. J Pediatr Gastroenterol Nutr 2018;66:122-127.

19. Lemoine C, Lokar J, McColley SA, et al. Cystic fibrosis and portal hypertension: Distal splenorenal shunt can prevent the need for future liver transplant. J Pediatr Surg 2019;54:1076-1082. 20**. Freeman AJ, Sellers ZM, Mazariegos G, et al. A Multidisciplinary Approach to Pretransplant and Posttransplant Management of Cystic Fibrosis-Associated Liver Disease. Liver Transpl 2019;25:640-657.

This multidisciplinary report presents clear recommandations for evaluation of liver or combined liver-lung transplantation of CF patients and post transplant management.

21. Bodewes FA, van der Doef HP, Houwen RH, Verkade HJ. Increase of Serum gammaGlutamyltransferase Associated With Development of Cirrhotic Cystic Fibrosis Liver Disease. J Pediatr Gastroenterol Nutr 2015;61:113-118.

22. Woodruff SA, Sontag MK, Accurso FJ, et al. Prevalence of elevated liver enzymes in children with cystic fibrosis diagnosed by newborn screen. J Cyst Fibros 2017;16:139-145.

23. Leung DH, Khan M, Minard CG, et al. Aspartate aminotransferase to platelet ratio and fibrosis-4 as biomarkers in biopsy-validated pediatric cystic fibrosis liver disease. Hepatology 2015;62:1576-1583.

24*. Ling SC, Ye W, Leung DH, et al. Liver Ultrasound Patterns in Children With Cystic Fibrosis Correlate With Noninvasive Tests of Liver Disease. J Pediatr Gastroenterol Nutr 2019;69:351-357. This study shows excellent discriminating ability of liver ultrasound for nodular liver versus normal liver in a multivariable model including GGT and APRI and and good ability to distinguish other US patterns.

25. Leung DH, Ye W, Molleston JP, et al. Baseline Ultrasound and Clinical Correlates in Children with Cystic Fibrosis. J Pediatr 2015;167:862-868 e862. 
26*. Sellers ZM, Lee LW, Barth RA, Milla C. New Algorithm for the Integration of Ultrasound Into Cystic Fibrosis Liver Disease Screening. J Pediatr Gastroenterol Nutr 2019;69:404-410.

This study report a combination of serum biomarkers can help direct which CF children should undergo US evaluation.

27. Aqul A, Jonas MM, Harney S, et al. Correlation of Transient Elastography With Severity of Cystic Fibrosis-related Liver Disease. J Pediatr Gastroenterol Nutr 2017;64:505-511.

28**. Gu J, Liu S, Du S, et al. Diagnostic value of MRI-PDFF for hepatic steatosis in patients with non-alcoholic fatty liver disease: a meta-analysis. Eur Radiol 2019;29:3564-3573.

This report confirms the excellent diagostic value of magnetic resonance imaging-proton density fat fraction for assessment of hepatic fat content.

29*. Lewindon PJ, Puertolas-Lopez MV, Ramm LE, et al. Accuracy of Transient Elastography Data Combined With APRI in Detection and Staging of Liver Disease in Pediatric Patients With Cystic Fibrosis. Clin Gastroenterol Hepatol 2019;17:2561-2569 e2565.

This a unique study showing that transient elastography combined with APRI differentiates between children with cystic fibrosis with mild-moderate fibrosis vs advanced fibrosis.

30. Gominon AL, Frison E, Hiriart JB, et al. Assessment of Liver Disease Progression in Cystic Fibrosis Using Transient Elastography. J Pediatr Gastroenterol Nutr 2018;66:455-460.

31. Klotter V, Gunchick C, Siemers E, et al. Assessment of pathologic increase in liver stiffness enables earlier diagnosis of CFLD: Results from a prospective longitudinal cohort study. PLoS One 2017;12:e0178784.

32. Franchi-Abella S, Corno L, Gonzales E, et al. Feasibility and Diagnostic Accuracy of Supersonic Shear-Wave Elastography for the Assessment of Liver Stiffness and Liver Fibrosis in Children: A Pilot Study of 96 Patients. Radiology 2016;278:554-562.

33**. Calvopina DA, Noble C, Weis A, et al. Supersonic shear-wave elastography and APRI for the detection and staging of liver disease in pediatric cystic fibrosis. J Cyst Fibros 2019. 
This is the first study evaluating the diagnostic accuracy of supersonic shear wave elastography in CF children, which is good in detecting CFLD and excellent for discriminating advanced disease. Combining liver stiffness assessment and APRI showed 14.8 times greater odds of accuraterly predicting CFLD.

34. Guglielmo FF, Venkatesh SK, Mitchell DG. Liver MR Elastography Technique and Image Interpretation: Pearls and Pitfalls. Radiographics 2019;39:1983-2002.

35. Schwimmer JB, Behling C, Angeles JE, et al. Magnetic resonance elastography measured shear stiffness as a biomarker of fibrosis in pediatric nonalcoholic fatty liver disease. Hepatology 2017;66:1474-1485.

36**. Lefebvre T, Wartelle-Bladou C, Wong P, et al. Prospective comparison of transient, point shear wave, and magnetic resonance elastography for staging liver fibrosis. Eur Radiol 2019;29:64776488.

This study shows that MRE has a higher diagnostic accuracy for discriminating early stages of fibrosis compared to TE or SWE in adults with viral or autoimmune chronic hepatitis.

37. Furlan A, Tublin ME, Yu L, et al. Comparison of 2D Shear Wave Elastography, Transient Elastography, and MR Elastography for the Diagnosis of Fibrosis in Patients With Nonalcoholic Fatty Liver Disease. AJR Am J Roentgenol 2020;214:W20-W26.

38**. Cardey J, Le Gall C, Michaud L, et al. Screening of esophageal varices in children using esophageal capsule endoscopy: a multicenter prospective study. Endoscopy 2019;51:10-17.

The first study to report the usefullness of esophageal capsule endoscopy for screening esophageal varices in children.

39*. Pai AK, Jonas MM, Fox VL. Esophageal Capsule Endoscopy in Children and Young Adults With Portal Hypertension. J Pediatr Gastroenterol Nutr 2019;69:641-647.

This study supports the use of esophageal capsule endoscopy for screening and surveillance of esophageal varices in children. 
40*. Kim DW, Yoon HM, Jung AY, et al. Diagnostic Performance of Ultrasound Elastography for Evaluating Portal Hypertension in Children: A Systematic Review and Meta-analysis. J Ultrasound Med 2019;38:747-759.

This systematic review reports the diagnostic performance of US elastography assessing spleen stiffness in evaluating portal hypertension in children.

41. Sintusek P, Siriporn N, Punpanich D, et al. Spleen and Liver Stiffness to Detect Esophageal Varices in Children with Biliary Atresia. J Pediatr Gastroenterol Nutr 2019;69:411-415.

42*. Sutton H, Fitzpatrick E, Davenport M, et al. Transient Elastography Measurements of Spleen Stiffness as a Predictor of Clinically Significant Varices in Children. J Pediatr Gastroenterol Nutr 2018;67:446-451.

This study shows that spleen stiffness measurement by TE can be used as a noninvasive screening tool for children with portal hypertension to stratify the risk of having clinically significant varices. 43*. Yokoyama S, Ishigami M, Honda T, et al. Spleen stiffness by 2-D shear wave elastography is the most accurate predictor of high-risk esophagogastric varices in children with biliary atresia. Hepatol Res 2019;49:1162-1168.

This study shows that spleen stiffness measurement by SWE can be used as a noninvasive screening tool for children with portal hypertension to stratify the risk of having high-risk esophagogastric varices.

44. Gana JC, Turner D, Mieli-Vergani G, et al. A clinical prediction rule and platelet count predict esophageal varices in children. Gastroenterology 2011;141:2009-2016.

45. Isted A, Grammatikopoulos T, Davenport M. Prediction of esophageal varices in biliary atresia: Derivation of the "varices prediction rule", a novel noninvasive predictor. J Pediatr Surg 2015;50:1734-1738. 


\section{Legends to figures}

Figure 1. 3D-MCRP showing focal stenosis of the left biliary duct (A). Correlation with Axial T2$\mathrm{WI}(B)$.

Figure 2. PDFF-sequence allowing detection and quantification of focal (ROI $2=48.9 \%$ ) and diffuse $($ ROI $1=14 \%)$ steatosis compared to subcutaneous fat $($ ROI $3=86.4 \%)($ A $)$ with excellent correlation on IN/OUT phases showing signal drop of peri portal area $(B$ and $C)$.

Figure 3. Hypersignal with focal capsular retraction on T2-WI and Diffusion sequence ( $A$ and $B$ ) correlated by the high precision of the MRE color mapping showing focal increase of the stiffness measurement $(C)$. 


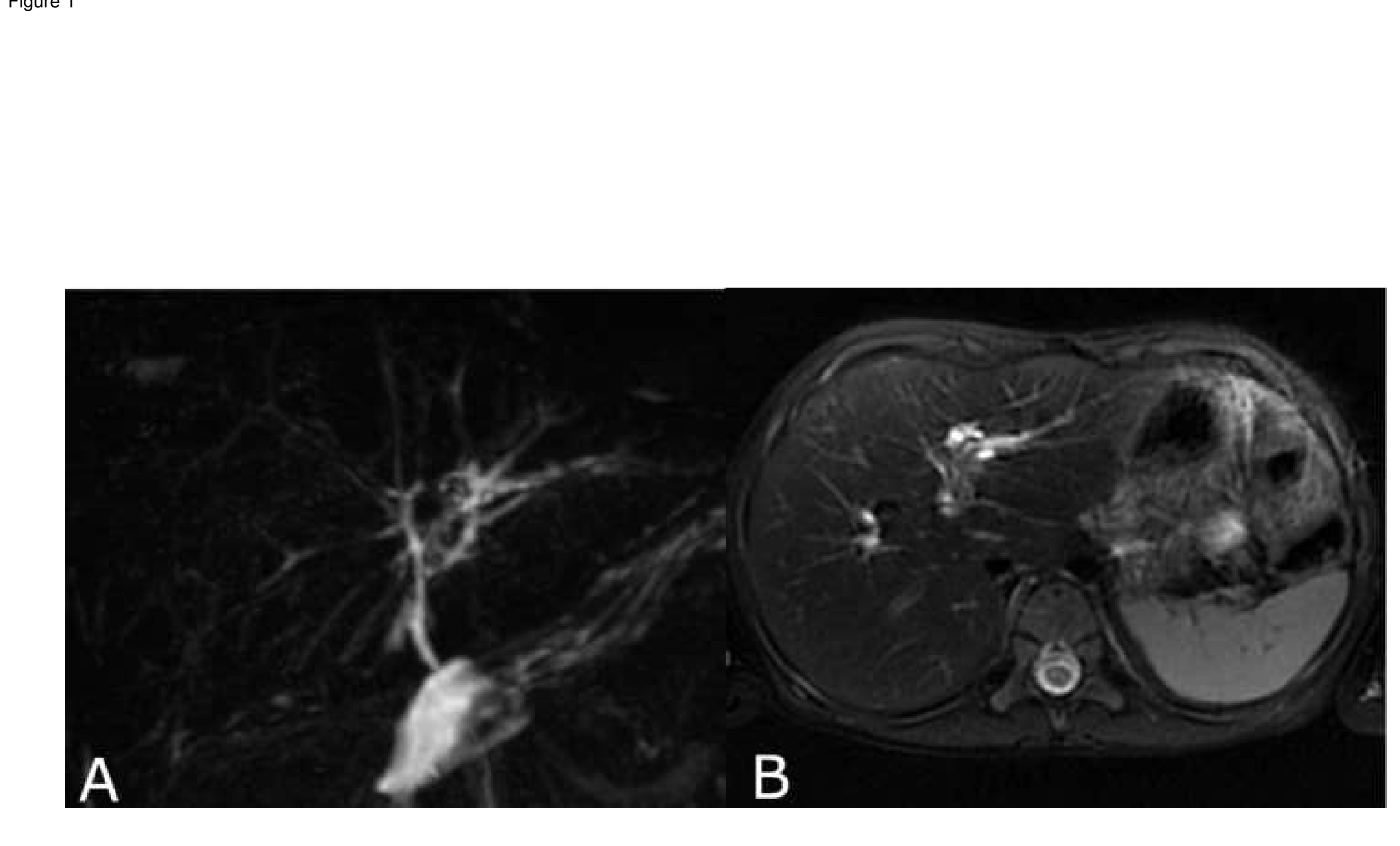

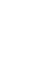
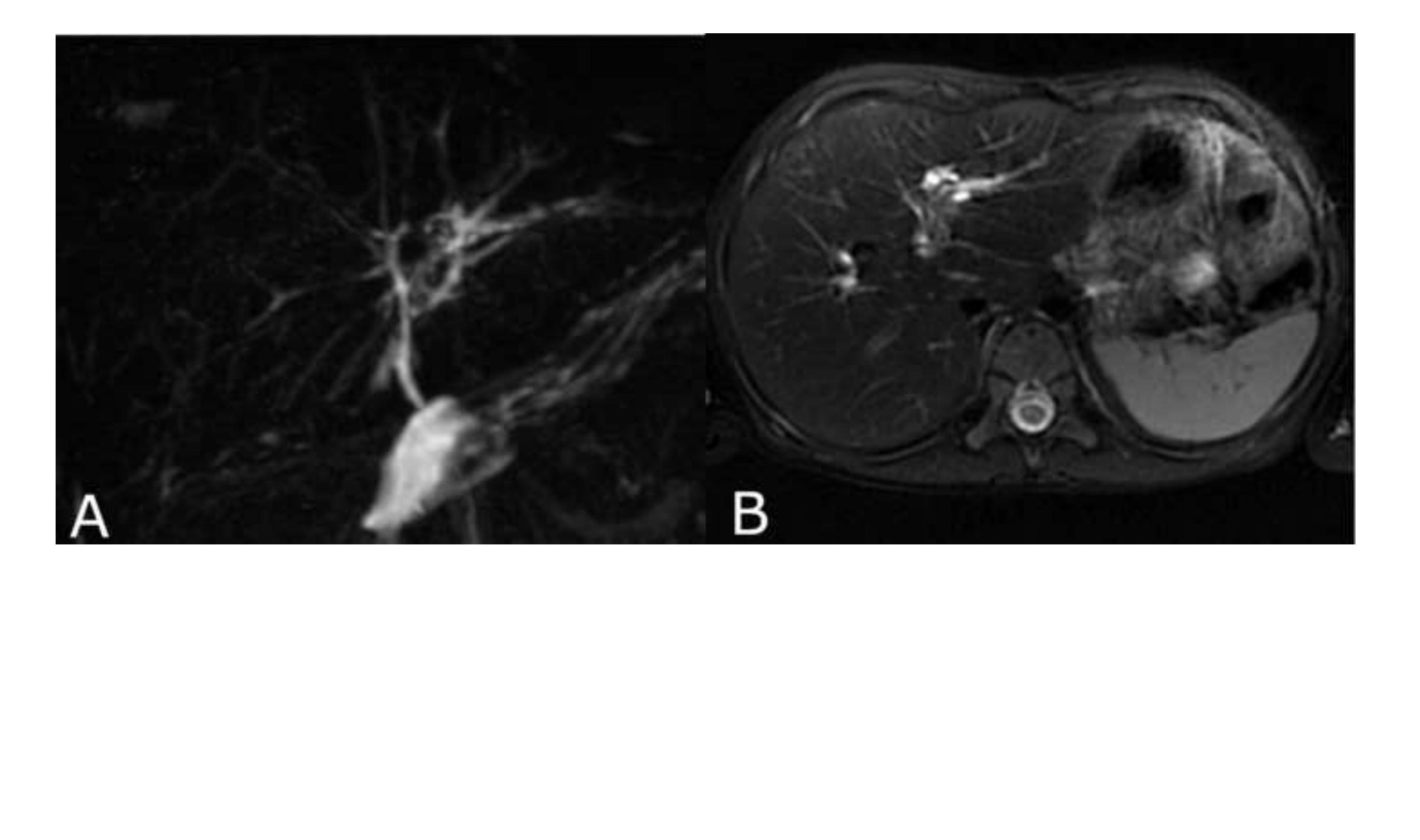


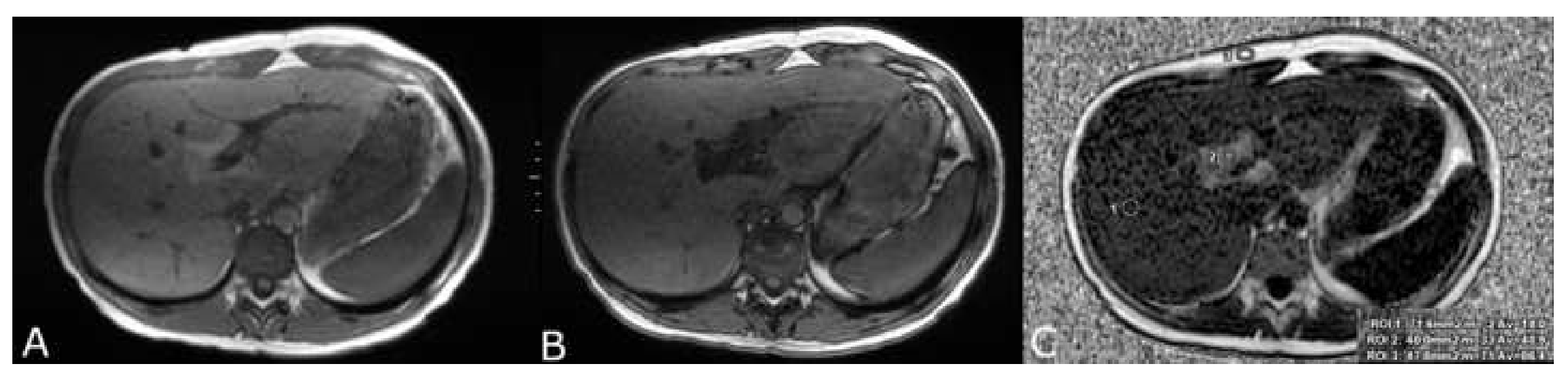




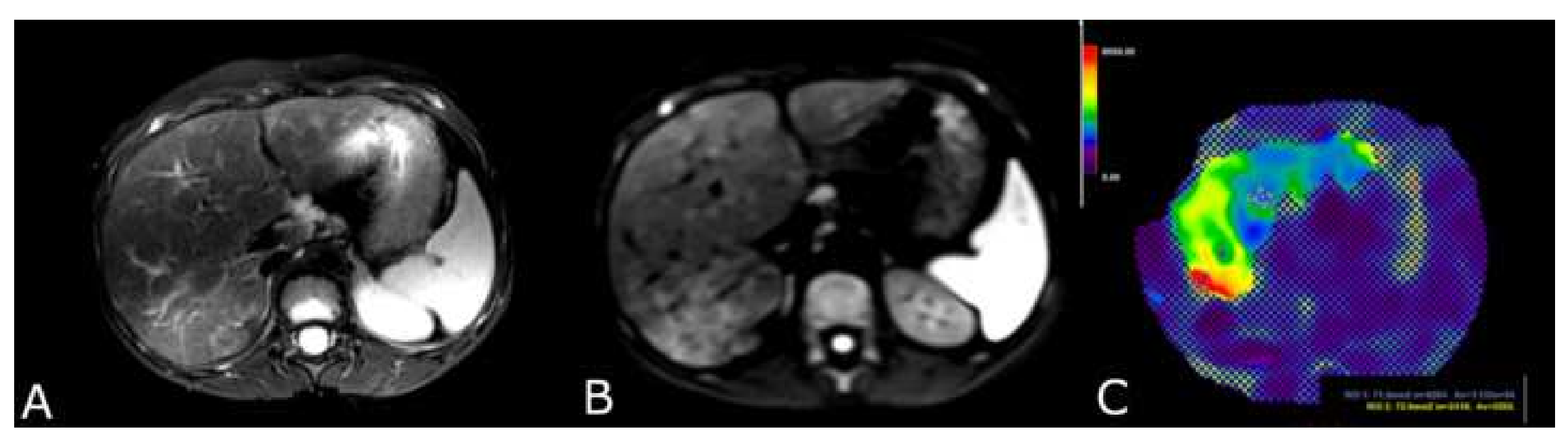

